\title{
Le développement du tibétain ancien -e- dans les dialectes occidentaux
}

The development of Old Tibetan-e-in Western dialects

\section{Guillaume Jacques}

\section{(2) OpenEdition \\ 1 Journals}

\section{Édition électronique}

URL : https://journals.openedition.org/emscat/1500

DOI : $10.4000 /$ emscat.1500

ISSN : 2101-0013

Éditeur

Centre d'Etudes Mongoles \& Sibériennes / École Pratique des Hautes Études

Référence électronique

Guillaume Jacques, "Le développement du tibétain ancien -e- dans les dialectes occidentaux », Études mongoles et sibériennes, centrasiatiques et tibétaines [En ligne], 40 | 2009, mis en ligne le 28 décembre 2009, consulté le 13 juillet 2021. URL : http://journals.openedition.org/emscat/1500 ; DOI : https:// doi.org/10.4000/emscat. 1500

Ce document a été généré automatiquement le 13 juillet 2021.

(c) Tous droits réservés 


\section{Le développement du tibétain ancien -e- dans les dialectes occidentaux}

The development of Old Tibetan-e-in Western dialects

Guillaume Jacques

1 L'écriture tibétaine, conçue au septième siècle de notre ère, représente la quasi-totalité des distinctions reconstructibles à partir des données dialectales modernes: rien n'autorise à affirmer qu'existaient en proto-tibétain (l'ancêtre commun de toutes les formes écrites et parlées de tibétain attestées) des distinctions qui ne seraient pas reflétées par l'écriture du tibétain ancien. Toutefois, certaines oppositions phonologiques attestées dans des dialectes périphériques, telles que l'existence d'une série d'uvulaires dans des dialectes de l'est, peuvent peut-être refléter une distinction proto-tibétaine non-attestée dans l'écriture (Sun 2003: 783, Suzuki 2008).

2 Le cas le plus discuté d'une distinction proto-tibétaine perdue dans la langue écrite mais préservée dans certains dialectes est celle de -ja- / -e- en tibétain de l'ouest, c'est à dire les dialectes Balti, Purik et Ladakhi. C'est Shafer (1941:19) qui a le premier attiré l'attention sur une série de correspondances où le -e- du tibétain ancien correspond soit à -ja-, soit à -e- dans les dialectes de l'ouest. Dans cet article, il cite trois exemples de -e- :: -ja- : lte-ba :: it fa 'nombril', legs-mo :: ljaymo ‘bon' et theg :: thjaq 'soulever'.

3 Son interprétation de cette correspondance est la suivante. Constatant l'absence en tibétain ancien de la médiane -j- derrière les initiales apicales (affriquées, occlusives, fricatives et les liquides, latérale et vibrante) et observant que tous ses exemples de -e:: -ya- apparaissent précisément derrière des initiales appartenant aux catégories suscitées il en conclut que le tibétain ancien a confondu *-ja- et *-e- dans le contexte après ces initiales, autrement dit un changement phonétique du type :

$4 \quad *_{\mathrm{ja}} \rightarrow \mathrm{e} /[$ +apicale]_ 
5 Les dialectes de l'ouest, n'ayant pas subi ce changement phonétique, auraient en revanche fidèlement préservé la distinction proto-tibétaine entre *ja et *e derrière dentale.

6 L'hypothèse de Shafer soulève toutefois un certain nombre de problèmes. En effet, les latérales du proto-tibétain subissent une série importante de changements phonétiques entre le proto-tibétain et le tibétain ancien (Michailovsky and Mazaudon 1994: 553, Jacques $2004: 4$ ). Dans les langues proches du tibétain comme le kurtöp, le monpa de Mtshosna et le tamang, le zh- du tibétain correspond le plus généralement à une latérale :

\begin{tabular}{|l|l|l|l|}
\hline tibétain & sens & proto-tibétain & kurtöp \\
\hline \hline zhing & champs & *ljin & Lley \\
\hline bzhi & quatre & *plji & ble \\
\hline \hline gzhu & arc & *klju & Llimi? \\
\hline
\end{tabular}

Tableau 1 : zh- provenant de laterals en tibétain

7 Le comparatisme suggère donc de façon claire que l'initiale translitérée zh-, une fricative sonore alvéolo-palatale [z], provient d'une latérale palatalisée. On peut donc proposer l'existence d'un changement phonétique ${ }^{*} \mathrm{lj}$ - $\rightarrow$ zh- entre proto-tibétain et tibétain classique. Or, d'après l'hypothèse de Shafer, le -e- d'un mot tel que legs 'bon' viendrait d'un proto-tibétain *ljaks en vertu de la correspondance avec le tibétain occidental. Toutefois, il apparaît que cette reconstruction n'est pas possible, car le résultat attendu serait *zhags en tibétain ancien, en vertu de la loi proposée ci-dessus. L'hypothèse de Shafer, basée sur des données internes au tibétain, n'est donc pas tenable.

8 Toutefois, les dialectes tibétains de l'est, grâce aux travaux de l'équipe dirigée par R. Bielmeier, sont désormais bien mieux connus qu'à l'époque où écrivait Shafer, et une réévaluation de son hypothèse est devenue possible au moyen de plus riches données.

9 Zemp (2006:101-103), offre une description détaillée de la double correspondance du e- dans tous les dialectes de l'ouest à sa disposition. Pour la simplification de l'exposé, nous limitons les données à trois des dialectes qu'il cite.

\begin{tabular}{|l|l|l|l|l|}
\hline Tibétain ancien & sens & Balti & Purik & Ladakhi (Nurla) \\
\hline reg & to begin & rjaxs & rjaqs & \\
\hline vtheng & to limp & thjay & thjay & \\
\hline ldeg & swinging up and down & ldjaqldjaq & ldjaqldjaq & \\
\hline gdeng & to trust, hope & & rdjay & \\
\hline bteg & to lift, go up, & & stjaq & \\
\hline
\end{tabular}




\begin{tabular}{|l|l|l|l|l|}
\hline reng & stiff & & rjanrjay & \\
\hline legs-mo & good & & djabmo & \\
\hline theg & to be able to lift & thjaq & stjaq & \\
\hline rtseg & to pile up & & Stsjaq & Stsak \\
\hline vdzegs & climb & & zdjaqs & dzak \\
\hline vkhengs & to get stiff & & khjays & \\
\hline
\end{tabular}

Tableau 2 : Correspondance e $:$. ja

10 Zemp (2006:105-106) rejette en revanche l'exemple lte ba :: Atja'nombril', proposé par Shafer, qu'il préfère à juste titre expliquer comme une contraction suite à la chute du b- intervocalique.

11 Le dernier exemple dans le tableau ci-dessus, comme le remarque Zemp (2006:103), réfute l'explication de Shafer: il prouve que la correspondance e :: ja existe aussi derrière vélaires. Une caractéristique commune de tous les exemples de ce tableau qui a toutefois échappé à Zemp est le fait que toutes les formes correspondantes en tibétain ancien sont des syllabes fermées en vélaire - $\mathrm{g}$ ou -ng.

12 Observons à présent les exemples où le e du tibétain ancien correspond à e en tibétain occidental :

\begin{tabular}{|l|l|l|l|l|}
\hline Tibétain ancien & sens & Balti & Purik & Ladakhi (Nurla) \\
\hline res & time & & res & \\
\hline mtheb po & thumb & & thepo & thebo \\
\hline ltem & full & & dtem & dtemdtem \\
\hline de & that & & de & de \\
\hline mdze & leprosy & & $z e$ & dze \\
\hline khyem & shovel & & khjem & \\
\hline
\end{tabular}

Tableau 3 : Correspondance e :.: e

13 Une comparaison entre ces deux tableaux permet une conclusion évidente : les formes du Tableau 2 ont une finale vélaire, tandis que celles du Tableau 3 sont en syllabe ouverte, ou ont une finale labiale ou dentale. Les deux correspondances e :: ja et e :: e sont donc manifestement en distribution complémentaire, et contrairement à ce que proposent Shafer et à Zemp, il est déraisonable de reconstruire deux voyelles ou diphtongues distinctes en proto-tibétain. Il est plus simple de poser le changement phonétique suivant : 
$*_{\mathrm{e}} \rightarrow \mathrm{ja}$ / _[vélaire]

dans les dialectes tibétains de l'ouest, qui seraient ici innovants, et non conservateurs comme le pensaient ces auteurs.

\section{BIBLIOGRAPHIE}

Jacques, G.

2004 The laterals in Tibetan, communication à Himalayan Languages Symposium, Bhoutan, Thimphu.

Michailovsky, B. and M. Mazaudon

1994 Preliminary notes on the languages of the Bumthang group, in Per Kvaerne (ed.) Tibetan Studies, Proceedings of the 6th seminar of the International Association for Tibetan Studies, Fagernes 1992, (Oslo, The Institute for Comparative Research in Human Culture), pp. 545-557.

Shafer, R.

1941 The Vocalism of Sino-Tibetan II, Journal of the American Oriental Society, 61, pp. 18-31.

Sun, J. T.-S.

2003 Phonological profile of Zhongu: A new Tibetan dialect of Northern Sichuan, Language and Linguistics, (Taipei, Academia Sinica), 4.4, pp. 769-836.

Suzuki, Hiroyuki

2008 Origin of non-Tibetan words in Tibetan dialects of the Ethnic Corridor in West Sichuan, International Symposium on 'Linguistic Substrata in Tibet', Osaka, National Museum of Ethnology 9-11 September.

Zemp, M.

2006 Synchronic and Diachronic Phonology of the Tibetan Dialect of Kargil, Lizentiatsarbeit (these de mastaire), Université de Berne, accessible à l'adresse : http://www.isw2.unibe.ch/tibet/

RESEARCH/Zemp_Purik.pdf

\section{RÉSUMÉS}

Cet article montre que la double correspondance e :: e et e :: ja entre le tibétain ancien et les dialectes occidentaux (ladakhi, balti, purik) ne reflète pas une distinction phonologique ancienne du proto-tibétain qui aurait été perdue en tibétain ancien et conservée dans ces dialectes.

This article shows that the double correspondence e :: e / e :: ja between Old Tibetan and modern Western dialects (Ladakhi, Balti, Purik) does not reflect an old phonological distinction from proto-Tibetan lost in old Tibetan and preserved in those dialects as was proposed in Shafer (1941). 
INDEX

Mots-clés : phonologie

Keywords : phonology

nomsmotscles Balti, Ladakhi, Purik, Tibétain

\section{AUTEUR}

\section{GUILLAUME JACQUES}

Guillaume Jacques est membre du CRLAO (CNRS). Sa recherche porte sur la description et l'étude historique des langues qianguiques (tangoute, rgyalrong et pumi). Il est l'auteur d'un ouvrage sur le tangoute paru en 2007 (Lincom Europa) et d'une monographie sur le japhug rgyalrong publiée en 2008 (Minzu chubanshe). 